\title{
Altered Gut Microbiota in Korean Children with Autism Spectrum Disorders
}

\author{
Sungji Ha ${ }^{1,+} \mathbb{D}$, Donghun Oh ${ }^{2,+}$, Sunghee Lee ${ }^{3} \mathbb{D}$, Jaewan Park ${ }^{3} \mathbb{D}$, Jaeun Ahn ${ }^{4} \mathbb{D}$, Sungku Choi ${ }^{3}$ \\ and Keun-Ah Cheon $4, *$ (D)
}

1 Department of Psychiatry, Institute of Behavioral Science in Medicine, Yonsei University College of Medicine, Seoul 03722, Korea; neuroscience79@gmail.com

2 Graduate School of Medicine, Yonsei University College of Medicine, Seoul 03722, Korea; dhun0329@gmail.com

3 Research Lab., Ildong Pharmaceutical Co., Ltd., Hwaseong 18449, Korea; slee@ildong.com (S.L.); pjw250@ildong.com (J.P.); alanchoi@ildong.com (S.C.)

4 Division of Child and Adolescent Psychiatry, Department of Psychiatry, Severance Children's Hospital, Institute of Behavioral Science in Medicine, Yonsei University College of Medicine, Seoul 03722, Korea; jaeun.ahn87@gmail.com

* Correspondence: kacheon@yuhs.ac; Tel.: +82-2-2228-1620

+ These authors contributed equally to this work.

check for updates

Citation: Ha, S.; Oh, D.; Lee, S.; Park, J.; Ahn, J.; Choi, S.; Cheon, K.-A. Altered Gut Microbiota in Korean Children with Autism Spectrum Disorders. Nutrients 2021, 13, 3300. https://doi.org/10.3390/nu13103300

Academic Editors: Jacopo Troisi and Alessio Fasano

Received: 7 September 2021

Accepted: 13 September 2021

Published: 22 September 2021

Publisher's Note: MDPI stays neutral with regard to jurisdictional claims in published maps and institutional affiliations.

Copyright: (C) 2021 by the authors Licensee MDPI, Basel, Switzerland. This article is an open access article distributed under the terms and conditions of the Creative Commons Attribution (CC BY) license (https:/ / creativecommons.org/licenses/by/ $4.0 /$ )

\begin{abstract}
Autism spectrum disorder (ASD) is a neurodevelopmental disorder characterized by social and behavioral impairments. Recent studies have suggested that gut microbiota play a critical role in ASD pathogenesis. Herein, we investigated the fecal microflora of Korean ASD children to determine gut microbiota profiles associated with ASD. Specifically, fecal samples were obtained from 54 children with ASD and 38 age-matched children exhibiting typical development. Systematic bioinformatic analysis revealed that the composition of gut microbiota differed between ASD and typically developing children (TDC). Moreover, the total amounts of short-chain fatty acids, metabolites produced by bacteria, were increased in ASD children. At the phylum level, we found a significant decrease in the relative Bacteroidetes abundance of the ASD group, whereas Actinobacteria abundance was significantly increased. Furthermore, we found significantly lower Bacteroides levels and higher Bifidobacterium levels in the ASD group than in the TDC group at the genus level. Functional analysis of the microbiota in ASD children predicted that several pathways, including genetic information processing and amino acid metabolism, can be associated with ASD pathogenesis. Although more research is needed to determine whether the differences between ASD and TDC are actually related to ASD pathogenesis, these results provide further evidence of altered gut microbiota in children with ASD, possibly providing new perspectives on the diagnosis and therapeutic approaches for ASD patients.
\end{abstract}

Keywords: autism spectrum disorder; microbiome; microbiota-gut-brain axis; Korean

\section{Introduction}

Autism spectrum disorder (ASD) is a neurodevelopmental disorder characterized by persistent social communication deficits, with restricted and repetitive patterns of behaviors, interests, or activities [1]. According to a recent report, ASD prevalence in 2016 was reported to occur in 18.5 per 1000 children aged 8 years in the United States [2]. In particular, ASD prevalence in South Korea was estimated to be $2.64 \%$, which had been attracting worldwide attention [3]. As ASD prevalence increases over time, the social burden also increases significantly. In fact, the cost of supporting an individual with ASD in a lifespan was approximately \$1.5-2.4 million in the United States and $10.92-1.5$ million in the United Kingdom [4].

Although the cause of ASD has yet to be identified, recent studies have reported that the gut microbiome can play a role in its pathogenesis [5]. Particularly, changes in 
the intestinal environment caused by the gut microbiota have been found to affect the production of signaling substances, consequently affecting mature brain functioning as well as prenatal and postnatal central nervous system (CNS) development [6]. This connection, which is called the microbiota-gut-brain axis, refers to the bidirectional communication pathway between gut bacteria and the CNS and is known to be associated with various processes, including neuroinflammation, stress axis activation, neurotransmission, bloodbrain-barrier (BBB) formation, myelination, microglia maturation, and neurotransmitter synthesis [7,8]. As an example, several researchers have confirmed that germ-free mice exhibited increased hippocampal neurogenesis, increased BBB permeability, and abnormal behaviors, such as decreased sociality and increased locomotor activity [9-12], showing that normal gut microbiota can modulate brain function and behavioral outcomes. Furthermore, Sharon et al. confirmed that microbiota colonization from ASD patients was sufficient to induce autistic behaviors in mice, and microbial metabolites were found to improve abnormal behaviors in an ASD mouse model [13].

Gastrointestinal (GI) problems, including abdominal pain, constipation, and diarrhea, are commonly observed in ASD patients [14-16]. Additionally, behavioral problems in ASD patients were related to GI disturbance severity, wherein ASD patients with GI disturbances showed lower social skills, higher anxiety, and more frequent aggressive behaviors than those without GI issues $[17,18]$. These associations between ASD and GI problems suggest a possible significant relationship between the gut microbiota and ASD.

Notably, several case-control studies have affirmed the possible roles of gut microbiota in ASD pathogenesis through the analysis of aberrant gut microbiota compositions in ASD patients. The most common findings were a decreasing trend in the Bacteroidetes to Firmicutes ratio [19-21] and a higher Clostridium abundance in ASD patients than in normal controls [22-24]. Despite these findings, there is little consensus on the phenotypic signature of the gut microbiome in ASD patients. One of the reasons for these inconsistent results is that the microbiome composition can be influenced by various factors, such as diet, lifestyle, and medical history [5]. In fact, it has been shown that the gut microbiota can be stably altered by dietary changes and exposure to xenobiotics, including antibiotics [25].

Although the role of the microbiome in ASD pathogenesis has not been identified, many interventional studies based on microbiome modulation have been conducted. Liu et al., for one, showed that the probiotic Lactobacillus plantarum PS128 (PS128) had beneficial effects on the opposition/defiance behaviors of boys with ASD [26]. Kang et al. also found that the microbiota transfer therapy improved GI and behavioral symptoms in ASD children, additionally confirming that these effects persisted two years later $[27,28]$. At present, these results should be interpreted carefully, but it is expected that microbiomebased interventions can be an alternative to help children with ASD in the future. Therefore, various basic studies reflecting each race and culture are required.

In the present study, we aimed to identify gut microbiota profiles associated with ASD through the intestinal microflora analysis of Korean children with ASD and children with typical development. Specifically, the fecal microbiota of each participant was analyzed through the V3-V4 regions of the 16S ribosomal RNA (rRNA) sequencing, and the main microbiome metabolites and short-chain fatty acids (SCFAs) were analyzed to understand the microbiota-gut-brain crosstalk.

\section{Materials and Methods}

\subsection{Participants and Study Design}

This study was approved by the Institutional Review Board of the Severance Hospital, Yonsei University College of Medicine (4-2018-0745). Sample collection began in October 2018, wherein participants who visited the Severance Children's Hospital agreed to serve as fecal donors, providing written informed consent and questionnaire data sheets. Specifically, fecal samples were collected from 54 ASD children (4-13 years of age; 43 male and 11 females) and 39 typically developing children (TDC) (4-9 years of age; 18 males and 21 females). One sample of a girl from the TDC group was excluded from the data analysis 
since the extracted DNA did not pass the quality control. ASD participants were diagnosed by a child and adolescent psychiatrist based on the Diagnostic and Statistical Manual of Mental Disorders, 5th Edition [1], which was supplemented by the Autism Diagnostic Observation Schedule-2 (ADOS-2), Autism Diagnostic Interview-Revised (ADI-R), and Social Responsiveness Scale (SRS) [29-31]. Meanwhile, participants in the TDC group were screened by a child and adolescent psychiatrist based on clinical examinations, including intelligence tests, SRS, and direct patient interviews. All participants were also restricted from taking antibiotics for 3 months and probiotic supplements for 15 days prior to fecal sample preparation.

\subsection{Sample Collection and DNA Extraction}

Fecal samples were individually collected by parents at each home using an in-house collection kit, which were immediately stored at $4{ }^{\circ} \mathrm{C}$ and transferred to the laboratory within $20 \mathrm{~h}$. Fecal samples (100 mg, wet weight) were then mixed with $200 \mu \mathrm{L}$ of phosphatebuffered saline (PBS; pH 7.4; 1:9; PBS: saline), and DNA was extracted using the QIAamp DNA Mini QIAcube Kit (\#51326, Qiagen, Hilden, Germany). The DNA concentration and quality were determined using $1 \%$ agarose gel electrophoresis and NanoDrop ND-1000 spectrophotometry (NanoDrop Technologies Inc., Wilmington, DE, USA). Afterwards, genomic DNA was stored at $-80^{\circ} \mathrm{C}$ before delivery to a commercial sequencing facility (Macrogen, Seoul, Korea).

\subsection{Next Generation Sequencing Processing}

Genomic DNA was amplified by polymerase chain reaction (PCR), which used a universal primer set $(341 \mathrm{~F}$ and $805 \mathrm{R})$ to target the V3-V4 region of the $16 \mathrm{~S}$ rRNA gene, also allowing the Illumina overhang adaptor, where the forward primer $\left(341 \mathrm{~F}, 5^{\prime}\right.$ TCGTCGGCAGCGTCAGATGTGTATAAGAGACAGCCTACGGGNGGCWGCAG-3'; the underlined sequence indicates the target region primer) and the reverse primer $\left(805 R, 5^{\prime}-\right.$ GTCTCGTGGGCTCGGAGATGTGTATAAGAGACAGGACTACHVGGGTATCTAATCC-3') are found, to be used [32]. DNA amplification was performed under the following conditions: initial denaturation at $95{ }^{\circ} \mathrm{C}$ for $3 \mathrm{~min}$, followed by 25 cycles of denaturation at $95{ }^{\circ} \mathrm{C}$ for $30 \mathrm{~s}$, primer annealing at $55{ }^{\circ} \mathrm{C}$ for $30 \mathrm{~s}$, and extension at $72{ }^{\circ} \mathrm{C}$ for $30 \mathrm{~s}$, with a final elongation at $72{ }^{\circ} \mathrm{C}$ for $5 \mathrm{~min}$. Secondary amplification for Illumina NexTera barcode attachment was then performed with the i5 forward primer $\left(5^{\prime}-\right.$ AATGATACGGCGACCACCGAGATCTACAC- XXXXXXXX-TCGTCGGCAGCGTC- $3^{\prime} ; X$ indicates the barcode region) and i7 reverse primer ( $5^{\prime}$-CAAGCA GAAGACGGCATACGAGATXXXXXXXX-GTCTCGTGGGCTCGG-3'). The amplification conditions were similar to those described above, with the exception of the amplification cycle, which was set to eight. PCR products were confirmed by $1 \%$ agarose gel electrophoresis and visualized using a Gel Doc system (BioRad, Hercules, CA, USA) afterwards.

Amplified products were purified using a CleanPCR kit (CleanNAc, Inc., Waddinxveen, The Netherlands), wherein equal concentrations of purified products were pooled, and short fragments (non-target products) were removed using CleanPCR (CleanNA, Inc., Waddinxveen, The Netherlands). DNA quality and product size were then assessed using a Bioanalyzer 2100 (Agilent, Palo Alto, CA, USA) with a DNA 7500 chip. Afterwards, mixed amplicons were pooled, and sequencing was carried out at Macrogen, Inc. (Seoul, Korea) using the Illumina MiSeq Sequencing system (Illumina, San Diego, CA, USA) following the manufacturer's instructions.

The raw Illumina read data for all samples were deposited in the European Bioinformatics Institute European Nucleotide Archive database under the accession number PRJEB45948.

\subsection{Bioinformatic Processing}

We obtained demultiplexed, paired-end reads from the MiSeq platform and imported them into the Quantitative Insights into Microbial Ecology 2 (QIIME2, ver. 2020.2.0) 
software pipeline using the FASTQ manifest protocol [33]. Primers in the raw sequences were trimmed using the Cutadapt software [34], and paired-end reads were merged using the VSEARCH merge pair plugin [35]. The resulting merged reads were then filtered to exclude low-quality reads based on a minimum quality score of q30, removing ambiguous base calls and all chimeric sequences by applying the vsearch uchime_ref plugin [36]. Following this, multiple high-quality sequence alignments were performed using MAFFT ver. 7, a method suitable for multiple alignments of a large number of short sequences [37]. Uninformative base positions derived from the lane mask were removed, and the resulting aligned sequences were used to generate a phylogenetic tree using FastTree 2.1.10 [38]. Additionally, the EzBioCloud database, which is complementary to QIIME 2, was used for taxonomic analysis based on $80 \%$ identity using the BLAST+ consensus taxonomy classifier $[39,40]$.

Afterwards, alpha and beta diversity metrics were extracted using the core-metricsphylogenetic plugin, which were based on the phylogenetic and non-phylogenetic trees. Species richness and evenness were then compared based on the Faith-phylogenetic diversity and operational taxonomic units (OTUs) and the Shannon index and pielou_e, respectively [41,42]. Lastly, the overall phylogenetic distance between the two groups was estimated using weighted UniFrac dissimilarity based on the phylogenetic tree [43].

\subsection{SCFA Analysis}

One gram of feces was dissolved in $8 \mathrm{~mL}$ of distilled water and homogenized using a vortex mixer for $5 \mathrm{~min}$. The solution was then centrifuged at $3273 \mathrm{~g}$ for $10 \mathrm{~min}$ at $25^{\circ} \mathrm{C}$. The resulting supernatant was filtered through a $0.2-\mu \mathrm{m}$ cellulose acetate/surfactant-free membrane filter, and $20 \mu \mathrm{L}$ was injected into a Waters e2695 HPLC system equipped with a Waters 2489 UV detector. Afterwards, chromatographic separation was conducted under isocratic elution conditions using a Concise coregel $87 \mathrm{H} 3$ column $(7.8 \times 300 \mathrm{~mm}, 9 \mu \mathrm{m})$ (Concise Separations, San Jose, CA, USA) and a mobile phase of $0.01 \mathrm{~N}$ sulfuric acid, with the detection wavelength set at $210 \mathrm{~nm}$. Other chromatographic conditions included a column oven temperature of $35^{\circ} \mathrm{C}$, a flow rate of $0.6 \mathrm{~mL} / \mathrm{min}$, and a run time of $65 \mathrm{~min}$. Following chromatographic separation, individual SCFAs (acetic acid, propionic acid, and butyric acid) were identified and quantified based on the known standard retention times and peak areas (HPLC grade, Sigma-Aldrich, St. Louis, MO, USA).

\subsection{Statistical Analysis}

The Kruskal-Wallis test with Tukey's multiple comparisons was used to determine significant bacterial variables among the groups with ASD and TDC in phylum and genus levels. Additionally, this test was used for ratio analysis of Bacteroidetes to Firmicutes. Linear discriminant analysis effect size (LEfSe) was used to explore the potential bacterial biomarkers associated with different groups [44]. This algorithm was used not only for bacterial biomarker discovery but also for determining the functional differences between ASD children and TDC.

Alpha diversity indices, including the Faith phylogenetic diversity, OTUs, Shannon index, and pielou_e, were calculated using the QIIME 2 plugin, and the Wilcoxon test was used to estimate alpha diversity differences between each category using $\mathrm{R}$ version 4.0.2. Spearman's rank tests calculated the correlation coefficients between SCFA and microbial richness, including Faith_pd and observed OTUs. For beta diversity, the significant distance difference among groups was assessed using permutation-based multivariate analysis of variance (PERMANOVA) with 10,000 replicates, which was plugged into QIIME 2. We analyzed the correlation between the relative bacterial abundances and Principal component 1 (PC 1) of the weighted UniFrac distance using Spearman correlation in R version 4.0.2. to determine the bacteria that have the most influence on the community structure.

Based on the marker gene data and the reference genome database, the functional composition of the metagenome was predicted using phylogenetic investigation of communities by reconstruction of unobserved states (PICRUSts). The sequences were aligned 
against Greengenes ver.13.5., and Operational Taxonomic Units (OTUs) were assigned at $97 \%$ identity. The OTU table was uploaded to normalize by copy number, followed by metagenome prediction, and finally categorized by KEGG function on the Galaxy interface (http:/ / huttenhower.sph.harvard.edu/galaxy, Galaxy Version 1.0.0). All descriptive statistics were processed using $\mathrm{R}$ version 4.0.2.

\section{Results}

\subsection{Demographic Characteristics}

In this study, we analyzed fecal samples from a total of 92 participants, including 54 ASD children and 38 TDC (Table 1). No statistically significant differences were observed in the mean ages of the two groups. However, the gender ratio, IQ, and SRS scores showed a significant difference between ASD and TDC groups (Table 1).

Table 1. Characteristics of ASD and TDC groups.

\begin{tabular}{cccc}
\hline & $\begin{array}{c}\text { ASD (N = 54), } \\
\text { Mean } \pm \text { SD }\end{array}$ & $\begin{array}{c}\text { TDC (N = 38), } \\
\text { Mean } \pm \text { SD }\end{array}$ & $p$ \\
\hline Gender $(n)$ & & & \\
Male & $79.6 \%(43)$ & $47.4 \%(18)$ & 0.001 \\
Female & $20.4 \%(11)$ & $52.6 \%(20)$ & 0.021 \\
Age (year) & $7.0 \pm 2.1$ & $6.0 \pm 1.7$ & 0.000 \\
IQ & $58.2 \pm 17.8$ & $103.0 \pm 14.1$ & 0.000 \\
SRS (total, T score) & $91.1 \pm 14.5$ & $43.0 \pm 8.0$ & \\
ADI-R (total) & $31.7 \pm 24.9$ & - & \\
ADOS-2 (total) & $17.6 \pm 15.4$ & - & \\
ASD, autism spectrum disorder; TDC, typical developing children; SD, standard deviation; IQ, intelligence \\
quotient; SRS, Social Responsiveness Scale; ADI-R, Autism Diagnosis Interview-Revised; ADOS-2, Autism \\
Diagnostic Observation Schedule-2.
\end{tabular}

\subsection{Microbial Profiling of the ASD and TDC}

We performed a microbial taxonomic analysis to compare the ASD and TDC groups. We found significant differences in the gut Bacteroidetes and Actinobacteria proportions, which are representative bacterial phyla, between the two groups. Specifically, significantly higher Actinobacteria and significantly lower Bacteroidetes levels were observed in the ASD group than in the TDC group $(p<0.05)$ (Figure 1a). At the genus level, increased relative Bifidobacterium and decreased relative Bacteroides abundances were also observed in the ASD group as compared to that in the TDC group $(p<0.05)$ (Figure $1 b)$. Furthermore, the Bacteroidetes to Firmicutes ratio was found to be significantly decreased in the ASD group $(p<0.05)$ (Figure 1c). Although no statistically significant differences were found at the species level, a decreasing tendency in Bacteroides vulgatus and Bacteroides dorei was also observed in the ASD group in comparison to the TDC group (Supplementary Figure S1). 


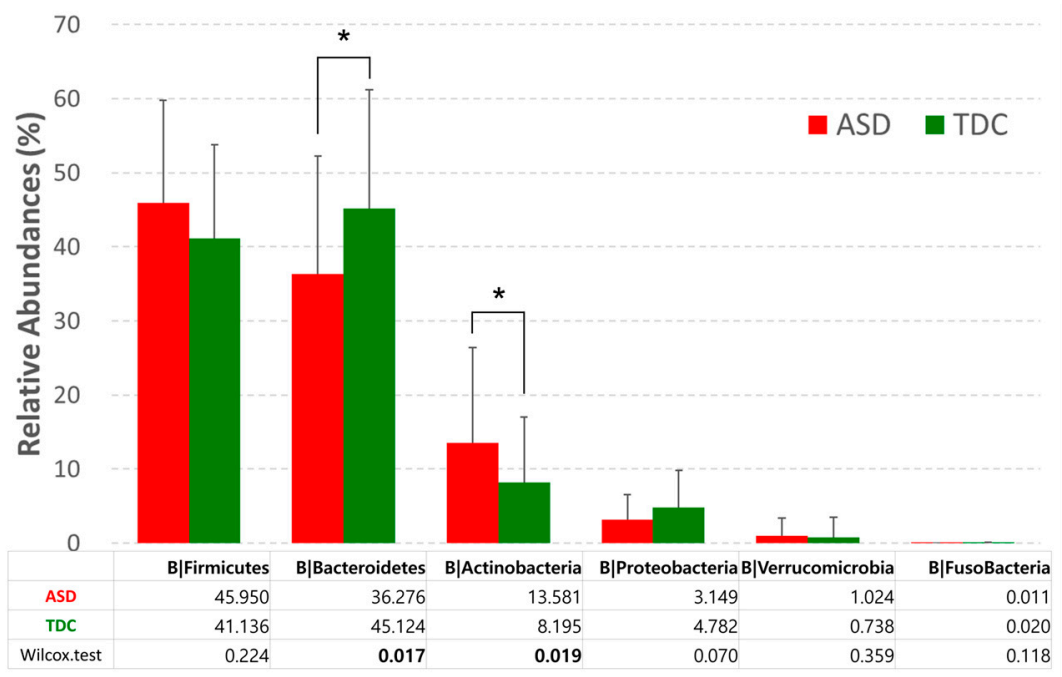

(a)

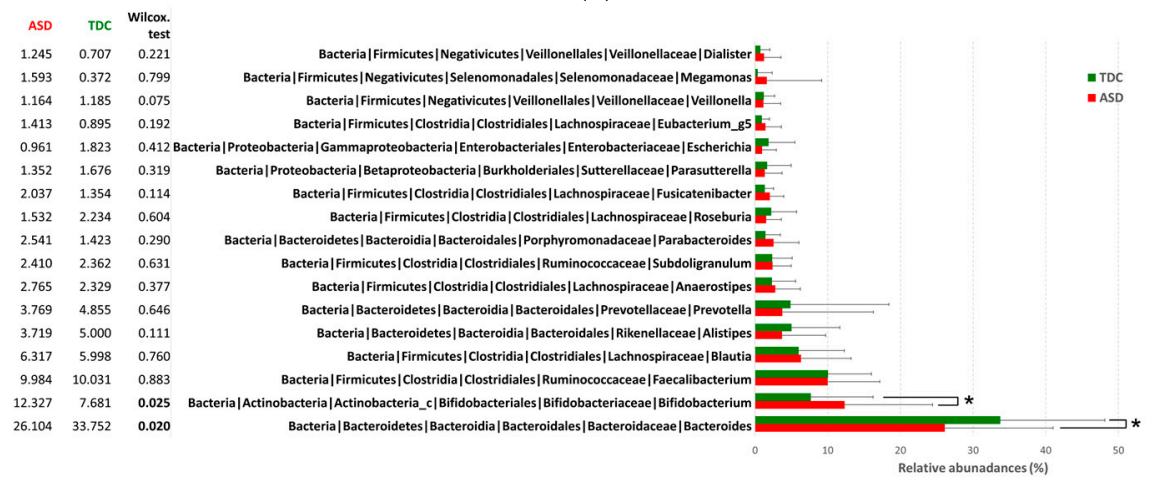

(b)



(c)

Figure 1. Microbial profiles at (a) the phylum and (b) the genus level and (c) Bacteroidetes/Firmicutes (B/F) ratio. The proportion of the bacterial composition is presented in tables $(\mathbf{a}, \mathbf{b})$. Error bars indicated standard deviation (S.D). Asterisk indicates significance between groups $\left({ }^{*} p<0.05\right)$. 
LEfSe analysis further confirmed these significant differences. Remarkably, a significant increase in the relative abundances of Actinobacteria_p, Actinobacteria_c, Bifidobacteriales_o, Bifidobacteriaceae_f, and Bifidobacterium_g (at the phylum to genus) as well as a significant reduction in Bacteroidetes_p, Bacteroidia_c, Bacteroidales_o, Bacteroidaceae_f, and Bacteroides_g (at the phylum to genus) were observed in the ASD group as compared to the TDC group (LDA score $>4.0, p<0.05$ ) (Figure 2).

When we further identified correlations between SRS scores and Bacteroides spp. or Bifidobacterium spp. in a group of ASD $(n=54)$, only Bacteroides spp. had a weak correlation with total SRS score ( $\mathrm{rs}=0.28, p<0.05$, data not shown). However, there was no significant correlation between the two bacteria and the five subscales of SRS, including social awareness, social cognition, social communication, social motivation, and autistic mannerism.

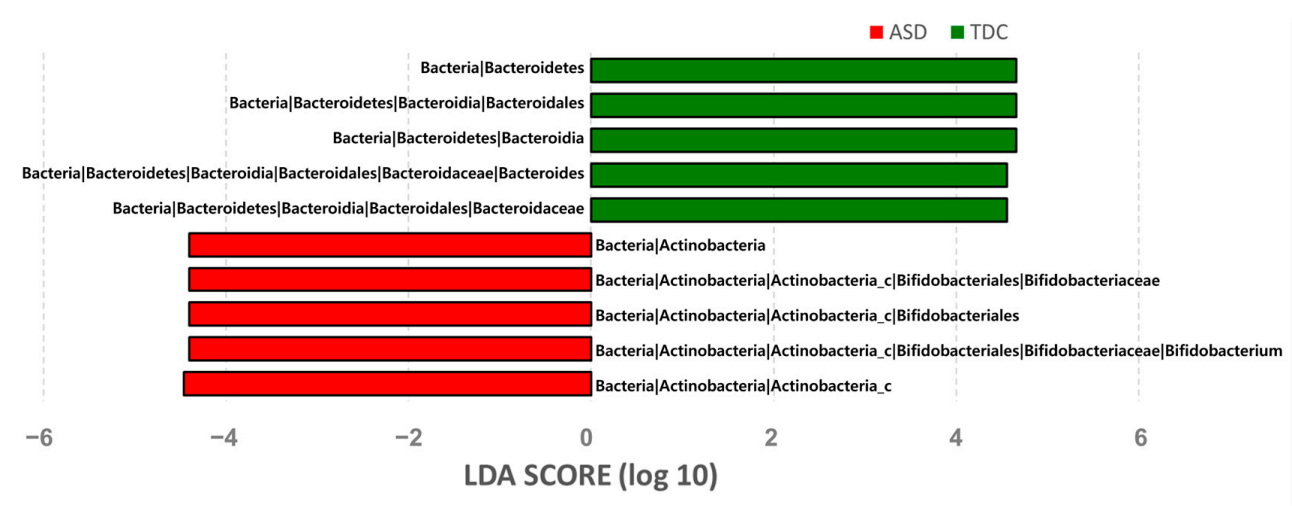

Figure 2. Spearman correlation between the relative bacterial abundances and Principal component 1 (PC 1) of weighted UniFrac analysis. LDA scores for discriminated bacterial taxa in the ASD and TDC.

\subsection{Microbial Diversitiy}

We analyzed the alpha diversity of ASD children and TDC based on richness, including Faith_pd and observed OTUs, and evenness, including the Shannon index and pielou_e. Alpha diversity analysis revealed no significant differences between ASD children and TDC, whereas beta diversity analysis based on the weighted UniFrac distances revealed that the bacterial community of ASD children was significantly different from that of TDC (PERMANOVA, pseudo-F $=2.649, p<0.05$ ) (Figure 3).

Moreover, principal component analysis was performed to determine which bacteria could effectively distinguish between the two groups. As a result, Bifidobacterium spp., which were abundantly present in the ASD group, including B. catenulatum and $B$. longum, were found to be the most influential factors that differentiated these two groups (Supplementary Figure S2).

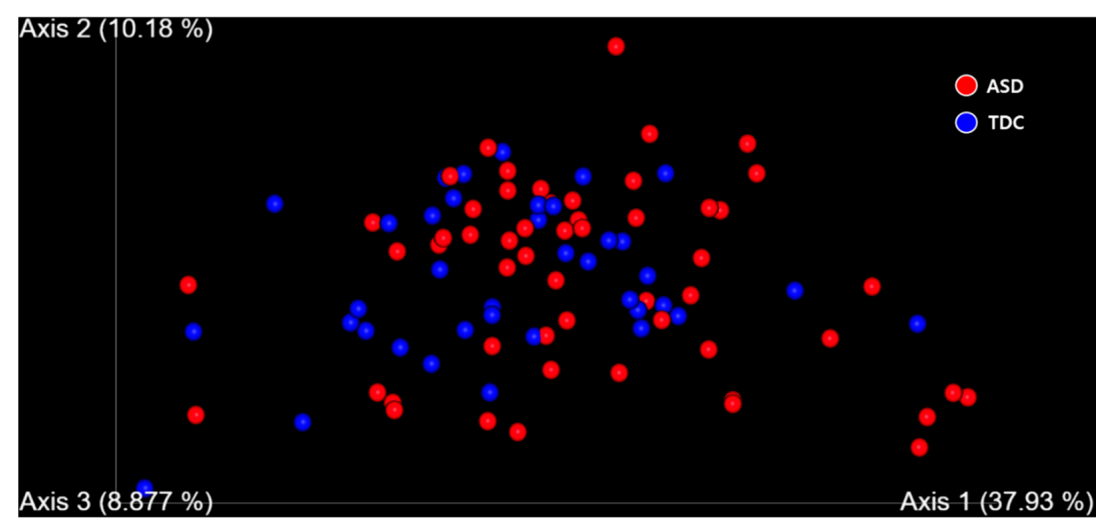

Figure 3. Beta diversity based on the weighted UniFrac distance in the ASD and TDC. Red and blue circles indicate ASD and TDC, respectively. 


\subsection{Functional Analysis}

The potential functions of the gut microbiota were predicted using PICRUSt analysis based on 16S rRNA sequences. Kyoto Encyclopedia of Genes and Genomes function analysis showed that genetic information processing and amino acid metabolism pathways were significantly higher in the ASD group than in the TDC group. In contrast, metabolism pathways, including carbohydrate and energy metabolism, were significantly higher in the TDC group (Figure 4).



Figure 4. Predicted metagenome function based on KEGG pathways analysis.

\subsection{Short-Chain Fatty Acid (SCFA) Analysis}

The presence of major SCFAs, acetic acid, propionic acid, and butyric acid, was measured in both groups, finding that the total amount of SCFAs was significantly higher in ASD children $(p<0.05)$. However, acetic acid, propionic acid, and butyric acid levels did not show any significant differences between the two groups (Figure 5a). Furthermore, no differences were found between the two groups on comparing the relative concentrations of SCFAs (Figure $5 b$ ).

To explore the relationship between SCFA production and microbial richness, including Faith_pd and observed OTUs, we performed a correlation analysis between them within the ASD and TDC groups. We found that higher microbial richness in the TDC group was attributed to a higher butyric acid production rate. In contrast, microbial richness in the ASD group did not affect the butyric acid production rate (Table 2).

Table 2. Spearman correlation analysis between SCFA and microbial richness. Spearman's rank tests calculated the correlation coefficients between SCFA and microbial richness, including Faith_pd and observed OTUs. The results with significant correlation $(p<0.05)$ are presented as an asterisk mark.

\begin{tabular}{ccccccccccc}
\hline \multicolumn{2}{c}{ Group } & \multicolumn{3}{c}{ Total (N = 92) } & \multicolumn{3}{c}{ ASD (N = 54) } & \multicolumn{3}{c}{ TDC (N = 38) } \\
\hline \multicolumn{2}{c}{ Relative SCFA } & AA & PA & BA & AA & PA & BA & AA & PA & BA \\
\hline \multirow{2}{*}{ Faith_pd } & $\mathrm{r}_{\mathrm{S}}$ & -0.185 & 0.000 & 0.318 & -0.006 & -0.188 & 0.216 & -0.428 & 0.264 & 0.490 \\
Observed & $p$ & 0.078 & 0.997 & $0.002^{*}$ & 0.964 & 0.174 & 0.117 & $0.008^{*}$ & 0.110 & $0.002^{*}$ \\
OTUs & $\mathrm{r}_{\mathrm{S}}$ & -0.101 & -0.011 & 0.253 & 0.083 & -0.154 & 0.103 & -0.319 & 0.169 & 0.438 \\
\hline
\end{tabular}

ASD, autism spectrum disorder; TDC, typical developing children; SCFA, short-chain fatty acid; AA, acetic acid; PA, propionic acid; BA, butyric acid. $\mathrm{r}_{\mathrm{s}}$ indicates correlation coefficient, ${ }^{*} p<0.05$. 


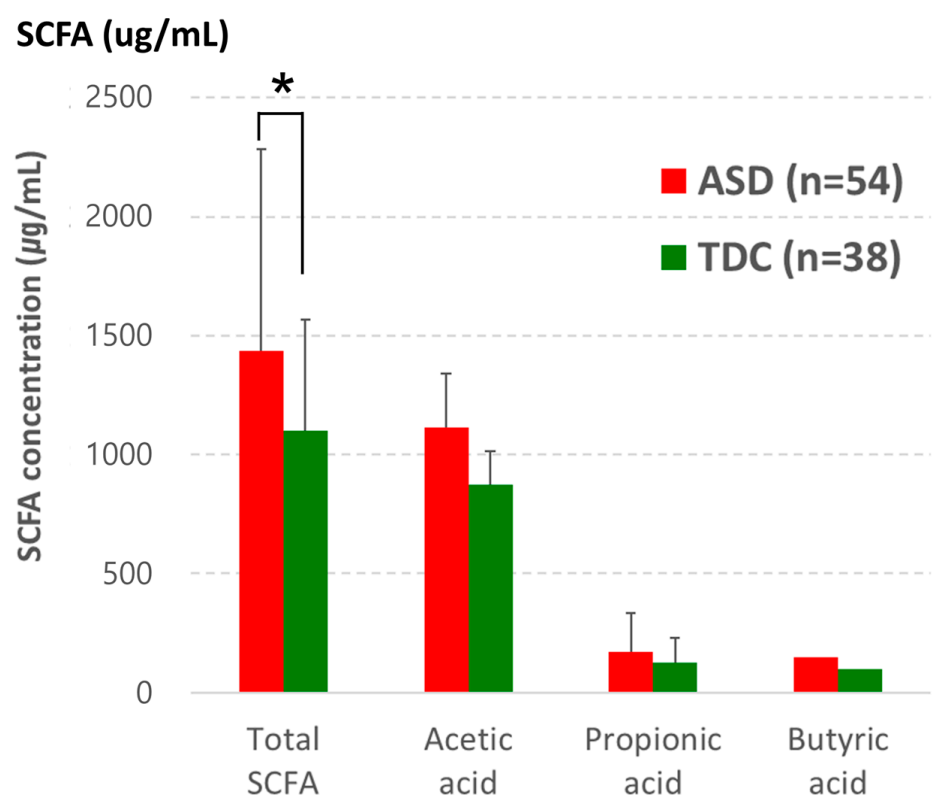

(a)

\section{SCFA (\%)}

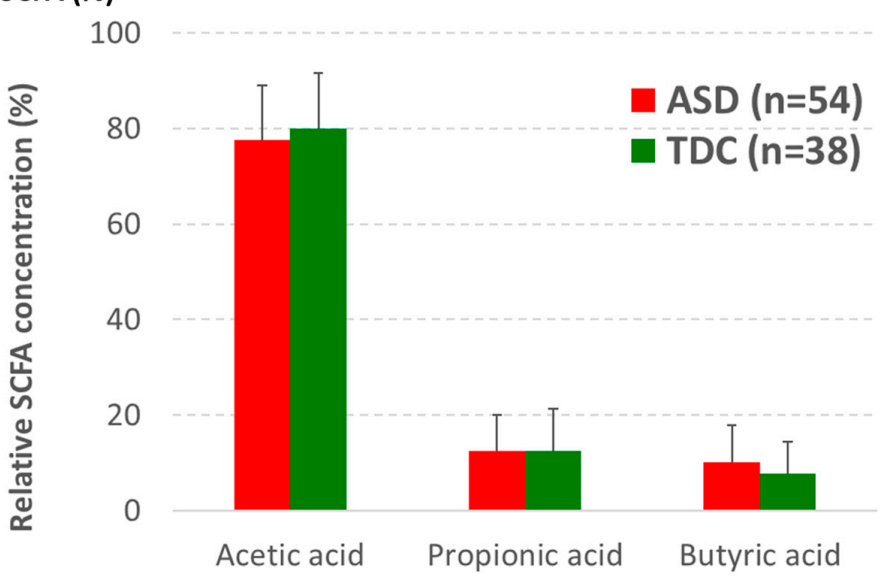

(b)

Figure 5. (a) Total SCFA and (b) the relative SCFA concentrations, including acetic acid and propionic acid butyric acid, in ASD and TDC. Error bars indicated standard deviation (SD). Asterisk indicates significance between groups $\left({ }^{*} p<0.05\right)$.

\section{Discussion}

In the present study, we characterized gut microbiota associated with ASD in Korean children. Using metagenomic analysis, we investigated the diversity of species in the samples. Among these, alpha diversity represents the richness and evenness of the microbiome in a single sample pool [45]. In the alpha diversity analysis of this study, we found no significant differences between the ASD and TDC groups, which was consistent with the results of several previous studies [21,46-48]. However, it should be noted that while other researchers have shown an increase in alpha diversity in the ASD group [49,50], some studies also showed a decrease in alpha diversity $[27,51,52]$. Beta diversity, on the other hand, refers to the dissimilarity between two microbial communities [45]. Differences between ASD and healthy control groups in beta diversity have been reported in previous studies, which was consistent with the current study's results $[21,27,49,52,53]$. Through principal component analysis, we confirmed that Bifidobacterium spp., which was significantly higher in the ASD group than the TDC group in the taxonomic analysis, was most influential factor that differentiated these two groups. 
To assess the composition of the gut microbiome in ASD, we conducted a microbial taxonomic analysis. As a result, we found a lower Bacteroidetes to Firmicutes ratio in the ASD group than in the TDC group due to a significant decrease in Bacteroidetes at the phylum level. A decreased Bacteroidetes to Firmicutes ratio in ASD patients has been consistently observed in previous studies [19-21] and has been associated with several inflammatory conditions, such as inflammatory bowel disease and obesity [54,55]. It is likely that the decreased proportion of Bacteroidetes in ASD may contribute to carbohydrate digestion and transport impairments. Williams et al., in particular, suggested that reduced intestinal gene expression involved in disaccharidase expression and hexose transporters found in ASD children was associated with microbial dysbiosis, possibly inducing GI disturbances, such as diarrhea and bloating [48].

Furthermore, we discovered that the relative abundance of the phylum Actinobacteria, including the genus Bifidobacterium, was significantly increased in the gut microbiota of the ASD group compared to that of the TDC group. Bifidobacterium, which is a common probiotic, promotes the production of different exopolysaccharides by acting as fermentable substrates for human gut bacteria [56]. However, in contrast to our findings, the proportion of Bifidobacterium has been reported to be reduced in ASD patients in previous studies $[49,57]$. Conversely, similar to our findings, Tomova et al. showed an increased Bifidobacterium abundance in ASD children, which was found to be decreased by probiotic supplementation. Liu et al. also demonstrated that vitamin A supplementation induced changes in Bacteroidetes/Bacteroidales as an ASD biomarker, consequently decreasing the proportion of Bifidobacterium [58].

In our study, the genus Bacteroides, belonging to the phylum Bacteroidetes, was found to be significantly decreased in ASD children as compared to that of TDC. Bacteroides species are one of the earliest colonizing and most numerically prominent microbial species in the gut microbiota [59]. Among them, Bacteroides fragilis is known to be effective in treating ASD-related symptoms. Hsiao et al., for one, confirmed that oral treatment using Bacteroides fragilis corrected gut permeability, altered microbial composition, and improved communicative, repetitive, sensorimotor, and anxiety-like behaviors in a maternal immuneactivation mouse model [60]. Although we did not find statistically significant differences between the two, we also confirmed that the two species belonging to the Bacteroides, Bacteroides vulgatus and Bacteroides dorei, were decreased in the ASD group, similar to Hsiao et al.'s study.

Since there is a lack of consistency in reported ASD microbiome studies, it is difficult to use the gut microbiome composition as a predictive biomarker for ASD. Despite this, it has been repeatedly confirmed that the gut microbiome composition in ASD patients differs significantly from that of normal developing controls, and we provided further evidence for these differences in the present study. In particular, we confirmed the possibility that Bacteroides reduction could be an important feature in the gut microbiome profile of Korean ASD children, and we believe that it can be used therapeutically in the future. We also identified a significant correlation between Bacteroides spp. and total SRS score, which was reported to be correlated with severity of ASD [31]. Contrary to our expectation, Bacteroides spp. and SRS score showed a weak positive correlation, and there was no significant correlation between the two bacteria and the five subscales of SRS. These results also suggest that Bacteroides spp. may be just involved in the pathogenesis of ASD. We speculated that Bacteroides spp. might be not dose-dependently related to the severity level of ASD, but more complex factors are likely to be involved in the symptom severity. To determine the possible roles of the gut microbiota, we predicted the functional profiles of microbial communities using PICRUSt analysis. As a result, we found that several pathways, including genetic information processing and amino acid metabolism pathway, were significantly higher in the ASD group than in the TDC group. In a systemic review by Liu et al., a PICRUSt analysis was also conducted using raw data from previous studies [45]. Similar with our results, pathways, such as "ABC transporter", "replication, recombination and repair proteins", "lysine biosynthesis", and "genetic information processing", showed 
high discriminative power in ASD patients in Strati et al.'s study [21]. Moreover, in two studies by Kang et al., "metabolism" and "amino acid metabolism" pathways were found to be commonly increased in ASD patients [27,51]. Regarding the metabolic pathway, host metabolism has been found to be regulated by metabolites derived from the intestinal microbes [61]. For example, dysregulated metabolism of free amino acids (FAA) has been observed in children with autism [49]. Interestingly, glutamate, which acts as a neurotransmitter in the brain, is also one of these identified FAAs and has been implicated in ASD pathophysiology [62]. Furthermore, Ming et al. demonstrated abnormal amino acid metabolism in ASD children by showing decreased levels of amino acids, such as glycine, serine, and glutamyl [63]. Although these functional analysis results provide a key clue to understanding the underlying mechanisms of ASD pathogenesis, more studies are needed to definitively determine how the gut microbiota affects ASD pathogenesis.

SCFAs are metabolites produced by the bacterial fermentation of complex polysaccharides and resistant starches, such as cellulose and pectin. Notably, major SCFAs, such as acetic acid, propionic acid, and butyric acid, account for more than $90 \%$ of SCFAs and are produced and absorbed in the colon $[64,65]$. Recent studies have suggested that SCFAs play a role in microbiota-gut-brain communication; however, the role of SCFAs in ASD pathology remains controversial [66]. In this study, although we found an increased total amount of SCFAs in ASD children, we could not find any significant difference in SCFA levels between the two groups. These results were similar to a study conducted by Wang et al. showing elevated fecal total SCFA concentrations in children with ASD [67]. Additionally, animal studies have also confirmed that SCFAs induce ASD-like behaviors and brain alterations $[68,69]$. On the other hand, another study reported that the total amount of SCFAs decreased in ASD, which was partly associated with increased probiotic use [15]. They further explained that altered fecal SCFA levels in ASD can be influenced by several factors, such as gut microbiome composition, food intake, gut transit time variabilities, and gut permeability [15]. Therefore, more research is required in ASD patients to elucidate the role of SCFAs in ASD. When we analyzed the correlation between microbial richness and major three SCFAs, we found that the higher the microbial richness in the TDC group, the higher the butyric acid (BA) production, but there was no correlation between microbial diversity and BA production in the ASD group. In general, microbial diversity means a healthy gut environment; however, it seems that high microbial diversity does not simply mean increased SCFA, beneficial substance in gut environments, especially in pathological conditions such as ASD. The reason that the increase in microbial richness in the ASD group did not affect the SCFA level is probably because the intestinal environment related to ASD influenced the SCFA production. Despite the findings of this study, several limitations were noted. First, although we observed significant differences in the microbiome composition between the two groups at the phylum and genus levels, this was not observed at the species level. Second, in SCFA analysis, no significant differences were found in each subtype of SCFAs, acetic acid, propionic acid, and butyric acid. It can be inferred that one of the reasons behind this was not only due to the insufficient number of participants but also because ASD participants with various severities were included. Third, many ASD children who participated in this study did not receive treatment despite complaints of chronic constipation symptoms, resulting in the differences in physical hardness observed during fecal sample collection. Lastly, a variety of factors not considered in this analysis may have influenced the results. Therefore, it is necessary to interpret whether the differences in microbiome composition between ASD and TDC observed in this study is related to the pathogenesis of ASD or due to other factors, such as differences in nutritional habits. In fact, it has already been known that ASD children are generally picky eaters who do not prefer vegetable intake [70]. Therefore, it will be necessary to conduct studies with a large number of children in a more homogenous group in the future. If an analysis is included in consideration of various factors affecting the gut microbiome composition, such as the severity of GI and ASD symptoms, medications, and diet habits, more infor- 
mation will be obtained to understand the relationship between ASD pathogenesis and intestinal microbes.

Nevertheless, to the best of our knowledge, this is the first study to show that the gut microbiome and their metabolites in Korean ASD children were significantly different when compared with TDC. Given our findings, we suggested that Bacteroides reduction was one of the characteristics observed in children with ASD in Korea, and it is expected that this finding can be used to help and treat ASD in this population in the future.

Supplementary Materials: The following are available online at https:/ /www.mdpi.com/article/10 $.3390 /$ nu13103300/s1, Figure S1: Microbial profiles at the at the species level, Figure S2: Spearman correlation between the relative bacterial abundances and Principal component 1 (PC 1) of weighted UniFrac analysis.

Author Contributions: Conceived and designed the study, S.H., D.O., S.L., S.C. and K.-A.C.; participants recruitment and evaluation, D.O., J.A. and K.-A.C.; fecal sample collection, S.H., S.L. and J.P.; clinical data collection and analysis, S.H., D.O. and K.-A.C.; microbiome data analysis, S.L. and J.P.; writing-original draft preparation, S.H., D.O. and S.L.; writing-review and editing, S.H., D.O., S.L., J.P., J.A., S.C. and K.-A.C.; supervision, K.-A.C.; funding acquisition, S.C. and K.-A.C. All authors have read and agreed to the published version of the manuscript.

Funding: This research was supported by a faculty research grant of Yonsei University College of Medicine for 2020 (6-2020-0232), the Basic Science Research Program through the National Research Foundation of Korea (NRF) grant funded by the Korea government (MSIT) (2021R1A2C2010913), and a grant funded by Ildong Pharmaceutical Co., Ltd., South Korea.

Institutional Review Board Statement: The study was conducted according to the guidelines of the Declaration of Helsinki and approved by the Institutional Review Board of Severance Hospital, Yonsei University College of Medicine (4-2018-0745).

Informed Consent Statement: Written informed consent was obtained from all participants and their parents involved in the study.

Data Availability Statement: The raw Illumina read data for all samples have been deposited in the European Bioinformatics Institute European Nucleotide Archive database, under study accession number PRJEB45948.

Acknowledgments: We appreciate all children and family members who gave their time and efforts to participate in the present study.

Conflicts of Interest: S.L., J.P. and S.C. are employees of Ildong Pharmaceutical Co., Ltd. Other authors declare no conflict of interest.

\section{References}

1. American Psychiatric Association. Diagnostic and Statistical Manual of Mental Disorders, 5th ed.; American Psychiatric Publishing: Washington, DC, USA, 2014.

2. Maenner, M.J.; Shaw, K.A.; Baio, J.; Washington, A.; Patrick, M.; DiRienzo, M.; Christensen, D.L.; Wiggins, L.D.; Pettygrove, S.; Andrews, J.G.; et al. Prevalence of autism spectrum disorder among children aged 8 years-Autism and developmental disabilities monitoring network, 11 sites, United States, 2016. MMWR. Surveill. Summ. 2020, 69, 1-12. [CrossRef] [PubMed]

3. Kim, Y.S.; Leventhal, B.L.; Koh, Y.-J.; Fombonne, E.; Laska, E.; Lim, E.-C.; Cheon, K.-A.; Kim, S.-J.; Lee, H.; Song, D.H.; et al. Prevalence of autism spectrum disorders in a total population sample. Am. J. Psychiatry 2011, 168, 904-912. [CrossRef] [PubMed]

4. Buescher, A.V.S.; Cidav, Z.; Knapp, M.; Mandell, D.S. Costs of autism spectrum disorders in the United Kingdom and the United States. JAMA Pediatr. 2014, 168, 721-728. [CrossRef] [PubMed]

5. Vuong, H.E.; Hsiao, E.Y. Emerging roles for the gut microbiome in autism spectrum disorder. Biol. Psychiatry 2017, 81, 411-423. [CrossRef]

6. Sharon, G.; Sampson, T.; Geschwind, D.H.; Mazmanian, S.K. The central nervous system and the gut microbiome. Cell 2016, 167, 915-932. [CrossRef]

7. Fung, T.C.; Olson, C.A.; Hsiao, E.Y. Interactions between the microbiota, immune and nervous systems in health and disease. Nat. Neurosci. 2017, 20, 145-155. [CrossRef]

8. Sherwin, E.; Dinan, T.G.; Cryan, J.F. Recent developments in understanding the role of the gut microbiota in brain health and disease. Ann. N. Y. Acad. Sci. 2017, 1420, 5-25. [CrossRef] 
9. Ogbonnaya, E.S.; Clarke, G.; Shanahan, F.; Dinan, T.; Cryan, J.F.; O'Leary, O. Adult hippocampal neurogenesis is regulated by the microbiome. Biol. Psychiatry 2015, 78, e7-e9. [CrossRef] [PubMed]

10. Braniste, V.; Al-Asmakh, M.; Kowal, C.; Anuar, F.; Abbaspour, A.; Tóth, M.; Korecka, A.; Bakocevic, N.; Ng, L.G.; Kundu, P.; et al. The gut microbiota influences blood-brain barrier permeability in mice. Sci. Transl. Med. 2014, 6, 263ra158. [CrossRef]

11. Arentsen, T.; Raith, H.; Qian, Y.; Forssberg, H.; Heijtz, R.D. Host microbiota modulates development of social preference in mice. Microb. Ecol. Health Dis. 2015, 26, 29719. [CrossRef]

12. Diaz Heijtz, R.; Wang, S.; Anuar, F.; Qian, Y.; Björkholm, B.; Samuelsson, A.; Hibberd, M.L.; Forssberg, H.; Pettersson, S. Normal gut microbiota modulates brain development and behavior. Proc. Natl. Acad. Sci. USA 2011, 108, 3047-3052. [CrossRef] [PubMed]

13. Sharon, G.; Cruz, N.J.; Kang, D.-W.; Gandal, M.J.; Wang, B.; Kim, Y.-M.; Zink, E.M.; Casey, C.P.; Taylor, B.C.; Lane, C.J.; et al. Human gut microbiota from autism spectrum disorder promote behavioral symptoms in mice. Cell 2019, 177, 1600-1618.e17. [CrossRef]

14. Buie, T.; Campbell, D.; Fuchs, G.J.; Furuta, G.T.; Levy, J.; Vandewater, J.; Whitaker, A.H.; Atkins, D.; Bauman, M.L.; Beaudet, A.L.; et al. Evaluation, diagnosis, and treatment of gastrointestinal disorders in individuals with asds: A consensus report. Pediatrics 2010, 125, S1-S18. [CrossRef] [PubMed]

15. Adams, J.B.; Johansen, L.J.; Powell, L.D.; Quig, D.; Rubin, R.A. Gastrointestinal flora and gastrointestinal status in children with autism-Comparisons to typical children and correlation with autism severity. BMC Gastroenterol. 2011, 11, 22. [CrossRef]

16. McElhanon, B.O.; McCracken, C.; Karpen, S.; Sharp, W.G. Gastrointestinal symptoms in autism spectrum disorder: A metaanalysis. Pediatrics 2014, 133, 872-883. [CrossRef]

17. Fulceri, F.; Morelli, M.; Santocchi, E.; Cena, H.; del Bianco, T.; Narzisi, A.; Calderoni, S.; Muratori, F. Gastrointestinal symptoms and behavioral problems in preschoolers with autism spectrum disorder. Dig. Liver Dis. 2016, 48, 248-254. [CrossRef] [PubMed]

18. Iovene, M.R.; Bombace, F.; Maresca, R.; Sapone, A.; Iardino, P.; Picardi, A.; Marotta, R.; Schiraldi, C.; Siniscalco, D.; Serra, N.; et al Intestinal dysbiosis and yeast isolation in stool of subjects with autism spectrum disorders. Mycopathologia 2016, 182, 349-363. [CrossRef]

19. Williams, B.L.; Hornig, M.; Parekh, T.; Lipkin, W.I.J.M. Application of novel PCR-based methods for detection, quantitation, and phylogenetic characterization of Sutterella species in intestinal biopsy samples from children with autism and gastrointestinal disturbances. mBio 2012, 3, e00261-11. [CrossRef] [PubMed]

20. Tomova, A.; Husarova, V.; Lakatosova, S.; Bakos, J.; Vlkova, B.; Babinska, K.; Ostatnikova, D. Gastrointestinal microbiota in children with autism in Slovakia. Physiol. Behav. 2015, 138, 179-187. [CrossRef]

21. Strati, F.; Cavalieri, D.; Albanese, D.; de Felice, C.; Donati, C.; Hayek, J.; Jousson, O.; Leoncini, S.; Renzi, D.; Calabrò, A.; et al New evidences on the altered gut microbiota in autism spectrum disorders. Microbiome 2017, 5, 1-11. [CrossRef] [PubMed]

22. Finegold, S.M.; Molitoris, D.; Song, Y.; Liu, C.; Vaisanen, M.; Bolte, E.; McTeague, M.; Sandler, R.; Wexler, H.; Marlowe, E.M.; et al. Gastrointestinal microflora studies in late-onset autism. Clin. Infect. Dis. 2002, 35, S6-S16. [CrossRef] [PubMed]

23. Song, Y.; Liu, C.; Finegold, S.M. Real-time PCR quantitation of clostridia in feces of autistic children. Appl. Environ. Microbiol. 2004, 70, 6459-6465. [CrossRef] [PubMed]

24. Alshammari, M.K.; AlKhulaifi, M.M.; Al Farraj, D.A.; Somily, A.; Albarrag, A.M. Incidence of clostridium perfringens and its toxin genes in the gut of children with autism spectrum disorder. Anaerobe 2020,61, 102114. [CrossRef]

25. Taguer, M.; Maurice, C.F. The complex interplay of diet, xenobiotics, and microbial metabolism in the gut: Implications for clinical outcomes. Clin. Pharmacol. Ther. 2016, 99, 588-599. [CrossRef]

26. Liu, Y.-W.; Liong, M.T.; Chung, Y.-C.E.; Huang, H.-Y.; Peng, W.-S.; Cheng, Y.-F.; Lin, Y.-S.; Wu, Y.-Y.; Tsai, Y.-C. Effects of Lactobacillus plantarum PS128 on children with autism spectrum disorder in Taiwan: A randomized, double-blind, placebocontrolled trial. Nutrients 2019, 11, 820. [CrossRef] [PubMed]

27. Kang, D.-W.; Adams, J.B.; Gregory, A.C.; Borody, T.; Chittick, L.; Fasano, A.; Khoruts, A.; Geis, E.; Maldonado, J.; McDonoughMeans, S.; et al. Microbiota transfer therapy alters gut ecosystem and improves gastrointestinal and autism symptoms: An open-label study. Microbiome 2017, 5, 10. [CrossRef] [PubMed]

28. Kang, D.-W.; Adams, J.B.; Coleman, D.M.; Pollard, E.L.; Maldonado, J.; McDonough-Means, S.; Caporaso, J.G.; Krajmalnik-Brown, R. Long-term benefit of microbiota transfer therapy on autism symptoms and gut microbiota. Sci. Rep. 2019, 9, 5821. [CrossRef]

29. Lord, C.; Rutter, M.; DiLavore, P.C.; Risi, S. Autism Diagnostic Observation Schedule—Generic, 2nd ed.; Western Psychological Corporation: Los Angeles, CA, USA, 2012.

30. Lord, C.; Rutter, M.; Le Couteur, A. Autism diagnostic interview-revised: A revised version of a diagnostic interview for caregivers of individuals with possible pervasive developmental disorders. J. Autism Dev. Disord. 1994, 24, 659-685. [CrossRef]

31. Cheon, K.-A.; Park, J.-I.; Koh, Y.-J.; Song, J.; Hong, H.-J.; Kim, Y.S.; Lim, E.-C.; Kwon, H.; Ha, M.; Lim, M.-H.; et al. The social responsiveness scale in relation to DSM IV and DSM5 ASD in Korean children. Autism Res. 2016, 9, 970-980. [CrossRef]

32. Muyzer, G.; de Waal, E.C.; Uitterlinden, A.G. Profiling of complex microbial populations by denaturing gradient gel electrophoresis analysis of polymerase chain reaction-amplified genes coding for 16S rRNA. Appl. Environ. Microbiol. 1993, 59, 695-700. [CrossRef]

33. Caporaso, J.G.; Kuczynski, J.; Stombaugh, J.; Bittinger, K.; Bushman, F.D.; Costello, E.K.; Fierer, N.; Peña, A.G.; Goodrich, J.K.; Gordon, J.I.; et al. QIIME allows analysis of high-throughput community sequencing data. Nat. Methods 2010, 7, 335-336. [CrossRef]

34. Martin, M. Cutadapt removes adapter sequences from high-throughput sequencing reads. EMBnet J. 2011, 17, 10-12. [CrossRef] 
35. Rognes, T.; Flouri, T.; Nichols, B.; Quince, C.; Mahé, F. VSEARCH: A versatile open source tool for metagenomics. PeerJ 2016, 4, e2584. [CrossRef] [PubMed]

36. Edgar, R.C.; Haas, B.J.; Clemente, J.C.; Quince, C.; Knight, R. UCHIME improves sensitivity and speed of chimera detection. Bioinformatics 2011, 27, 2194-2200. [CrossRef] [PubMed]

37. Katoh, K.; Misawa, K.; Kuma, K.; Miyata, T. MAFFT: A novel method for rapid multiple sequence alignment based on fast Fourier transform. Nucleic Acids Res. 2002, 30, 3059-3066. [CrossRef] [PubMed]

38. Price, M.N.; Dehal, P.S.; Arkin, A.P. FastTree: Computing large minimum evolution trees with profiles instead of a distance matrix. Mol. Biol. Evol. 2009, 26, 1641-1650. [CrossRef] [PubMed]

39. Camacho, C.; Coulouris, G.; Avagyan, V.; Ma, N.; Papadopoulos, J.S.; Bealer, K.; Madden, T.L. BLAST+: Architecture and applications. BMC Bioinform. 2009, 10, 421. [CrossRef]

40. Yoon, S.-H.; Ha, S.-M.; Kwon, S.; Lim, J.; Kim, Y.; Seo, H.; Chun, J. Introducing EzBioCloud: A taxonomically united database of 16S rRNA gene sequences and whole-genome assemblies. Int. J. Syst. Evol. Microbiol. 2017, 67, 1613-1617. [CrossRef] [PubMed]

41. Faith, D.P. Conservation evaluation and phylogenetic diversity. Biol. Conserv. 1992, 61, 1-10. [CrossRef]

42. Spellerberg, I.F.; Fedor, P.J. A tribute to Claude Shannon (1916-2001) and a plea for more rigorous use of species richness, species diversity and the 'Shannon-Wiener' index. Glob. Ecol. Biogeogr. 2003, 12, 177-179. [CrossRef]

43. Hamady, M.; Lozupone, C.A.; Knight, R.A. Fast UniFrac: Facilitating high-throughput phylogenetic analyses of microbial communities including analysis of pyrosequencing and PhyloChip data. ISME J. 2009, 4, 17-27. [CrossRef] [PubMed]

44. Segata, N.; Izard, J.; Waldron, L.; Gevers, D.; Miropolsky, L.; Garrett, W.S.; Huttenhower, C. Metagenomic biomarker discovery and explanation. Genome Biol. 2011, 12, R60. [CrossRef]

45. Liu, F.; Li, J.; Wu, F.; Zheng, H.; Peng, Q.; Zhou, H. Altered composition and function of intestinal microbiota in autism spectrum disorders: A systematic review. Transl. Psychiatry 2019, 9, 1-13. [CrossRef]

46. Gondalia, S.V.; Palombo, E.A.; Knowles, S.R.; Cox, S.B.; Meyer, D.; Austin, D.W. Molecular characterisation of gastro-intestinal microbiota of children with autism (with and without gastrointestinal dysfunction) and their neurotypical siblings. Autism Res. 2012, 5, 419-427. [CrossRef] [PubMed]

47. Kushak, R.I.; Winter, H.S.; Buie, T.M.; Cox, S.B.; Phillips, C.D.; Ward, N.L. Analysis of the duodenal microbiome in autistic individuals: Association with carbohydrate digestion. J. Pediatric Gastroenterol. Nutr. 2017, 64, e110-e116. [CrossRef] [PubMed]

48. Williams, B.L.; Hornig, M.; Buie, T.; Bauman, M.L.; Paik, M.C.; Wick, I.; Bennett, A.; Jabado, O.; Hirschberg, D.L.; Lipkin, W.I. Impaired carbohydrate digestion and transport and mucosal dysbiosis in the intestines of children with autism and gastrointestinal disturbances. PLoS ONE 2011, 6, e24585. [CrossRef] [PubMed]

49. De Angelis, M.; Piccolo, M.; Vannini, L.; Siragusa, S.; de Giacomo, A.; Serrazzanetti, D.I.; Cristofori, F.; Guerzoni, M.E.; Gobbetti, M.; Francavilla, R. Fecal microbiota and metabolome of children with autism and pervasive developmental disorder not otherwise specified. PLoS ONE 2013, 8, e76993. [CrossRef]

50. Finegold, S.M.; Dowd, S.; Gontcharova, V.; Liu, C.; Henley, K.E.; Wolcott, R.D.; Youn, E.; Summanen, P.H.; Granpeesheh, D.; Dixon, D.; et al. Pyrosequencing study of fecal microflora of autistic and control children. Anaerobe 2010, 16, 444-453. [CrossRef]

51. Kang, D.-W.; Park, J.G.; Ilhan, Z.E.; Wallstrom, G.; LaBaer, J.; Adams, J.B.; Krajmalnik-Brown, R. Reduced incidence of prevotella and other fermenters in intestinal microflora of autistic children. PLoS ONE 2013, 8, e68322. [CrossRef] [PubMed]

52. Kang, D.-W.; Ilhan, Z.E.; Isern, N.G.; Hoyt, D.W.; Howsmon, D.P.; Shaffer, M.; Lozupone, C.A.; Hahn, J.; Adams, J.B.; KrajmalnikBrown, R. Differences in fecal microbial metabolites and microbiota of children with autism spectrum disorders. Anaerobe 2018, 49, 121-131. [CrossRef]

53. Coretti, L.; Paparo, L.; Riccio, M.P.; Amato, F.; Cuomo, M.; Natale, A.; Borrelli, L.; Corrado, G.; de Caro, C.; Comegna, M.; et al. Gut microbiota features in young children with autism spectrum disorders. Front. Microbiol. 2018, 9, 3146. [CrossRef] [PubMed]

54. Macfabe, D.F.; Cain, D.P.; Rodriguez-Capote, K.; Franklin, A.E.; Hoffman, J.E.; Boon, F.; Taylor, A.R.; Kavaliers, M.; Ossenkopp, K.-P. Neurobiological effects of intraventricular propionic acid in rats: Possible role of short chain fatty acids on the pathogenesis and characteristics of autism spectrum disorders. Behav. Brain Res. 2007, 176, 149-169. [CrossRef] [PubMed]

55. Turnbaugh, P.J.; Bäckhed, F.; Fulton, L.; Gordon, J.I. Diet-induced obesity is linked to marked but reversible alterations in the mouse distal gut microbiome. Cell Host Microbe 2008, 3, 213-223. [CrossRef] [PubMed]

56. Salazar, N.; Gueimonde, M.; Hernández-Barranco, A.M.; Ruas-Madiedo, P.; de los Reyes-Gavilán, C.G. Exopolysaccharides produced by intestinal bifidobacterium strains act as fermentable substrates for human intestinal bacteria. Appl. Environ. Microbiol. 2008, 74, 4737-4745. [CrossRef]

57. Wang, L.; Christophersen, C.T.; Sorich, M.J.; Gerber, J.P.; Angley, M.T.; Conlon, M.A. Low relative abundances of the mucolytic bacterium Akkermansia muciniphila and Bifidobacterium spp. in feces of children with autism. Appl. Environ. Microbiol. 2011, 77, 6718-6721. [CrossRef]

58. Liu, J.; Liu, X.; Xiong, X.-Q.; Yang, T.; Cui, T.; Hou, N.-L.; Lai, X.; Liu, S.; Guo, M.; Liang, X.-H.; et al. Effect of vitamin A supplementation on gut microbiota in children with autism spectrum disorders-A pilot study. BMC Microbiol. 2017, 17, 1-14. [CrossRef]

59. Könönen, E.; Jousimies-Somer, H.; Asikainen, S. Relationship between oral gram-negative anaerobic bacteria in saliva of the mother and the colonization of her edentulous infant. Oral Microbiol. Immunol. 1992, 7, 273-276. [CrossRef] [PubMed] 
60. Hsiao, E.Y.; McBride, S.W.; Hsien, S.; Sharon, G.; Hyde, E.R.; McCue, T.; Codelli, J.A.; Chow, J.; Reisman, S.E.; Petrosino, J.F.; et al. Microbiota modulate behavioral and physiological abnormalities associated with neurodevelopmental disorders. Cell 2013, 155, 1451-1463. [CrossRef] [PubMed]

61. Ding, H.T.; Taur, Y.; Walkup, J.T. Gut microbiota and autism: Key concepts and findings. J. Autism Dev. Disord. 2017, 47, 480-489. [CrossRef]

62. Shimmura, C.; Suda, S.; Tsuchiya, K.; Hashimoto, K.; Ohno, K.; Matsuzaki, H.; Iwata, K.; Matsumoto, K.; Wakuda, T.; Kameno, Y.; et al. Alteration of plasma glutamate and glutamine levels in children with high-functioning autism. PLoS ONE 2011, 6, e25340. [CrossRef]

63. Ming, X.; Stein, T.P.; Barnes, V.; Rhodes, N.; Guo, L. Metabolic perturbance in autism spectrum disorders: A metabolomics study. J. Proteome Res. 2012, 11, 5856-5862. [CrossRef]

64. Topping, D.L.; Clifton, P.M. Short-chain fatty acids and human colonic function: Roles of Resistant starch and nonstarch polysaccharides. Physiol. Rev. 2001, 81, 1031-1064. [CrossRef]

65. Kim, K.N.; Yao, Y.; Ju, S.Y. Short chain fatty acids and fecal microbiota abundance in humans with obesity: A systematic review and meta-analysis. Nutrients 2019, 11, 2512. [CrossRef]

66. Dalile, B.; van Oudenhove, L.; Vervliet, B.; Verbeke, K. The role of short-chain fatty acids in microbiota-gut-brain communication. Nat. Rev. Gastroenterol. Hepatol. 2019, 16, 461-478. [CrossRef] [PubMed]

67. Wang, L.; Christophersen, C.T.; Sorich, M.J.; Gerber, J.P.; Angley, M.T.; Conlon, M.A. Elevated fecal short chain fatty acid and ammonia concentrations in children with autism spectrum disorder. Dig. Dis. Sci. 2012, 57, 2096-2102. [CrossRef] [PubMed]

68. Kratsman, N.; Getselter, D.; Elliott, E. Sodium butyrate attenuates social behavior deficits and modifies the transcription of inhibitory/excitatory genes in the frontal cortex of an autism model. Neuropharmacology 2016, 102, 136-145. [CrossRef] [PubMed]

69. Macfabe, D.F. Enteric short-chain fatty acids: Microbial messengers of metabolism, mitochondria, and mind: Implications in autism spectrum disorders. Microb. Ecol. Health Dis. 2015, 26, 28177. [CrossRef] [PubMed]

70. Tomova, A.; Soltys, K.; Kemenyova, P.; Karhanek, M.; Babinska, K. The influence of food intake specificity in children with autism on gut microbiota. Int. J. Mol. Sci. 2020, 21, 2797. [CrossRef] 